\title{
Les relations stratégiques Chine/Pakistan
}

Swaran SINGH (éd.), China-Pakistan Strategic Cooperation : Indian

Perspectives, New Delhi, Manohar / Centre de sciences humaines, 2007, $405 \mathrm{p}$.

\section{Gilles Boquérat}

\section{OpenEdition Journals}

Édition électronique

URL : http://journals.openedition.org/transcontinentales/924

DOI : 10.4000/transcontinentales.924

ISBN : 978-2-7351-1561-7

ISSN : 1775-397X

Éditeur

Editions de la maison des sciences de l'homme

\section{Édition imprimée}

Date de publication : 30 juin 2008

Pagination : 154-156

ISBN : 978-2-200-92511-6

ISSN : 1950-1684

\section{Référence électronique}

Gilles Boquérat, "Les relations stratégiques Chine/Pakistan », Transcontinentales [En ligne], 6 | 2008, document 13, mis en ligne le 06 avril 2011, consulté le 23 septembre 2020. URL : http://

journals.openedition.org/transcontinentales/924 ; DOI : https://doi.org/10.4000/transcontinentales. 924 
espaces ruraux en de multiples enclaves discontinues et exiguës, les rendant difficiles d'accès. L'édification de "murs" à partir de 2002 n'a fait qu'amplifier la tendance à l'isolement des campagnes. Des enquêtes sociologiques systématiques sur la formation et le profil des élites du monde rural auraient permis de documenter la thèse du «repli sur le local» et de faire émerger d'éventuelles recompositions identitaires en cours.

L'auteur préfère ici porter son attention sur les répertoires et modalités d'action d'une ONG de développement, et souligner les décalages éventuels existant entre discours et pratiques des acteurs. La partie consacrée à l'organisation du processus décisionnel interne à l'association est particulièrement éclairante à ce sujet : alors que PARC se présente comme œuvrant à l'implication des "communautés locales " et à la décentralisation, l'analyse fait, au contraire, ressortir un monde très hiérarchique centré autour de la seule autorité du "chef fondateur». C. Abu Sada pose d'ailleurs fort à propos la question de la pérennité de PARC. L'étude contribue aussi à la réflexion sur la "société civile» dans le monde arabe. En la matière - même si certains développements théoriques donnent l'impression de "passages obligés»-, les conclusions de l'auteur apparaissent stimulantes : elle qui estime que "l'usage du concept de société civile reste délicat dans sa version homogène et unifiée» (p. 233), démontre qu'il n'y a pas une, mais des sociétés civiles en Palestine qui, chacune, ont leur propre conception de la construction de l'État et du politique.

D'autres passages de l'ouvrage laissent cependant une impression de flou ou $\mathrm{d}^{\prime}$ inabouti au lecteur. Ainsi l'auteur indique-t-elle, à plusieurs reprises, qu'à l'arrivée de l'Autorité palestinienne, il a été question de transformer PARC en ministère de l'Agriculture. Mais ni la teneur des débats, ni les enjeux en la matière, ni même les raisons qui ont conduit à l'avortement du projet ne sont traités. Or, sans aucun doute s'agit-il là d'un moment "fondateur» pour les acteurs en présence, annonciateur des relations de pouvoir à venir. Cette remarque vaut aussi pour la partie consacrée à l'étude des liens entre l'ONG et son parti fondateur, dont le traitement, trop rapide et parfois teinté d'idéologie, laisse le lecteur sur sa faim.

Au final, malgré quelques imperfections, la publication de l'ouvrage de C. Abu Sada mérite d'être soulignée, tant le terrain palestinien peut constituer une sorte de laboratoire des rapports entre le secteur associatif et un État en devenir.

Aude Signoles, maître de conférences en science politique, associée à l'Institut d'études de l'Islam et des sociétés du monde musulman (IISMM) de I'EHESS, Paris

\section{Les relations stratégiques Chine/Pakistan}

\section{Swaran SingH (éd.),}

China-Pakistan Strategic Cooperation

Indian Perspectives, New Delhi,

Manohar / Centre de sciences humaines, 2007, $405 \mathrm{p}$

Il y a probablement peu d'amitiés que les décideurs pakistanais en matière de politique étrangère chérissent autant que celle de la Chine. Exemple parmi d'autres de cette attention toute particulière, l'Institut d'études stratégiques d'Islamabad a ouvert dans ses locaux en 2007 un centre d'études spécifiquement axé sur la Chine. Autre illustration de cette relation "à l'épreuve du temps", la présence croissante de citoyens chinois sur le sol pakistanais. Cet ouvrage, placé sous la direction de Swaran Singh, qui enseigne à l'université Jawaharlal 
Nehru et fut pendant plusieurs années affilié au Centre de sciences humaines de New Delhi, entreprend d'explorer la peu banale relation sino-pakistanaise. Il y avait dans les années 1950 peu de similarités idéologiques entre la République islamique alliée des États-Unis et la Chine maoïste. Sonika Gupta (The Role of Political Culture) argue toutefois qu'il y avait des similarités de culture politique sous la forme d'une interprétation réaliste du recours à la force et du rôle important joué par les militaires dans la construction de l'état et dans le processus de décision. Mais surtout il y eut stratégiquement la même volonté de circonscrire les ambitions indiennes (Satyabrat Sinha, China in Pakistan's Security Perceptions). Pour les dirigeants pakistanais, c'était l'intégrité même du pays qui aurait été menacée par l'Inde et tout contrepoids extérieur était précieux. Pour Pékin, il s'agissait de ne pas abandonner l'Asie du Sud à une zone d'influence exclusivement indienne. Sur cette base, non seulement une coopération stratégique se développa, mais aussi prospéra et survécut à la fin de la guerre froide et au rapprochement sino-indien observé au cours de la décennie écoulée. Investis du rôle de liant, les Indiens sont donc les premiers concernés par l'intensité d'une relation bilatérale qui à la fois les intrigue et les agace.

L'ouvrage est divisé en trois parties : les politiques et les perceptions réciproques, les coopérations stratégiques et en matière de défense, et les fondements de l'engagement stratégique. Si la relation avec la Chine est particulièrement prisée au Pakistan, elle n'en demeure pas moins intrinsèquement inégale, la dépendance étant surtout pakistanaise (Anindyo J. Majumdar, The Changing Imperatives). Si Islamabad sollicite avec une belle constance le soutien de la Chine, cette dernière, à l'instar de l'évolution des positions prises sur la question du Cachemire, ou encore au moment du conflit de Kargil (1999), a démontré qu'elle entendait garder sa liberté de jugement (Srikanth Kondappalli, Pakistan in China's Security Perception). Mais au moins, pour Islamabad, Pékin n'a jamais pris parti pour l'ennemi indien, à l'inverse par exemple des Américains. Courtisée, la Chine y trouve aussi des avantages: un passage vers le golfe Persique et le monde musulman, et un moyen de prévenir un "hégémonisme régional ». La coopération dans les domaines sensibles du nucléaire, de la technologie balistique et plus généralement du transfert d'armement fait l'objet d'un traitement approfondi où l'accent est inévitablement mis sur les aspects proliférationnistes et en contravention avec les accords internationaux de cette coopération. Un partenariat dont la dimension maritime est ici soulignée en reprenant la thèse d'une collusion pakistanaise à un encerclement naval de l'Inde auquel aspirerait Pékin en s'appuyant également sur le Myanmar (Vijay Sakhuja, Naval Cooperation; W. Lawrence S. Prabhakar, The Maritime Convergence).

La prégnance du stratégique est telle qu'elle déborde sur la relation économique, à commencer par la part significative des ventes $d^{\prime}$ armements chinois (Madhu Bhalla, Geopolitics of Economic Relations; Aparna Kher, Pakistan in China's Arms Trade). La Chine est souvent associée à deux grands projets qui, sans surprise, combinent intérêts stratégiques et commerciaux: le port de Gwadar, inauguré en 2007, presque trente ans après l'ouverture de la route du Karakorum, le seul lien terrestre qui doit d'ailleurs être aménagé dans les années à venir afin de permettre un trafic plus important (Virendra Sahai Verma, 
The Karakorum Highway). Le transit entre le Xinjiang et le nord du Pakistan peut aussi avoir des effets moins recherchés par Pékin lorsqu'il s'agit du soutien d'une militance islamiste aux séparatistes ouïgours (Abanti Bhattacharya, The Xinjiang Factor).

On peut seulement regretter que le délai entre la remise des contributions et la publication n'ait pas été plus court : cet intervalle souvent important dans les travaux collectifs, est plus encore dommageable sur des questions d'actualité. Face au parti pris d'avoir un grand nombre d'auteurs (18), des coupes auraient aussi été souhaitables afin d'éviter nombre de redites. On aurait également aimé que la relation bilatérale soit parfois replacée dans une perspective plus globale. Il est souvent écrit par exemple que le Pakistan représente pour la Chine un point d'entrée dans le monde musulman, mais sans donner des manifestations concrètes de cette attente. Cet ouvrage n'en offre pas moins un panorama quasi exhaustif des multiples facettes de la coopération stratégique sino-pakistanaise telle qu'elle est perçue par les analystes indiens. Il comble à cet égard un vide.

Gilles Boquérat, chercheur résident à l'Institut d'études stratégiques d'Islamabad, chercheur associé au Centre d'études de l'Inde et de l'Asie du Sud (CNRS-EHESS), Paris
Globalisation et espaces locaux

Margarita Estrada IguínIZ et Pascal Labazée (coord.), Globalización y localidad: espacios, actores, movilidades e identidades, Mexico, Publicaciones de la Casa Chata, Centro de Investigación y Estudios Superiores en Antropología social (CIESAS) et Institut de recherche

pour le développement, 2007, 625 p.

Cet ouvrage, épais volume de plus de 600 pages, explore les liens entre la globalisation et les espaces locaux. La plupart des vingt et quelque contributions portent sur le Mexique, le plus souvent sur l'État de Guanajuato, mais quelques-unes présentent des études de cas réalisées au Brésil, en Argentine ou à Porto Rico, voire en Inde ou au Mali. Dans l'ensemble, disons-le d'emblée, ce choix emporte l'adhésion: la confrontation d'observations centrées sur un terrain particulier à des éclairages, même rapides, sur d'autres parties du monde permet effectivement de mieux saisir la dimension globale des évolutions en cours, tout en soulignant les particularités locales.

L'ouvrage est composé d'un prologue, d'une introduction et de quatre sections, autour des espaces productifs, des acteurs sociaux, des mobilités et du rapport entre travail et identité. La distribution des textes pose parfois question. À première lecture, la reconfiguration des espaces ruraux du Veracruz semble être aussi bien le pendant des recompositions industrielles des villes du Guanajuato qu'une affaire de migrations; les textes portant sur les migrants sont classés parfois du côté de la réflexion sur les acteurs sociaux, parfois de la description des mobilités. Les auteurs préviennent cependant la critique dès l'introduction : leur objectif est précisément de montrer l'interdépendance des quatre thèmes. Le lecteur est effectivement convié à reconstituer depuis plusieurs angles de vue, en 